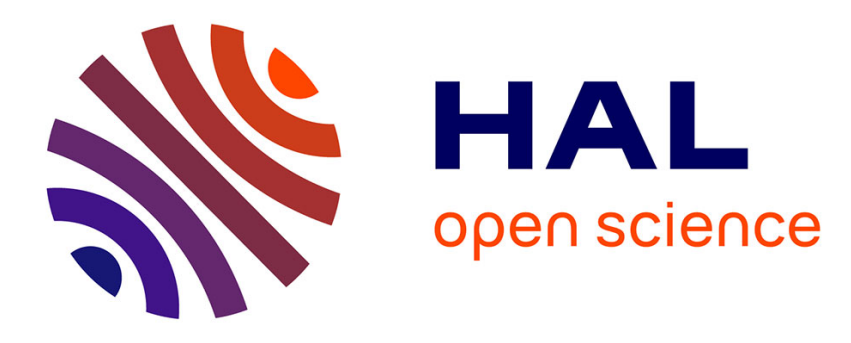

\title{
DYNAMICAL EFFECTS IN NEUTRON OPTICS AND SPIN INTERACTIONS
}

\author{
M. Forte
}

\section{To cite this version:}

M. Forte. DYNAMICAL EFFECTS IN NEUTRON OPTICS AND SPIN INTERACTIONS. Journal de Physique Colloques, 1984, 45 (C3), pp.C3-249-C3-253. 10.1051/jphyscol:1984342 . jpa-00224059

\section{HAL Id: jpa-00224059 https://hal.science/jpa-00224059}

Submitted on 1 Jan 1984

HAL is a multi-disciplinary open access archive for the deposit and dissemination of scientific research documents, whether they are published or not. The documents may come from teaching and research institutions in France or abroad, or from public or private research centers.
L'archive ouverte pluridisciplinaire HAL, est destinée au dépôt et à la diffusion de documents scientifiques de niveau recherche, publiés ou non, émanant des établissements d'enseignement et de recherche français ou étrangers, des laboratoires publics ou privés. 
JOURNAL DE PHYSIQUE

Colloque C3, supplément au n³, Tome 45, mars 1984

page C3-249

\title{
DYNAMICAL EFFECTS IN NEUTRON OPTICS AND SPIN INTERACTIONS
}

\author{
M. Forte \\ Physics Division, Joint Research Centre - Ispra Establishment, \\ 21020 Ispra ( Va), Italy
}

Résumé - On étudie la production d'effets d'optique neutronique qui sont sensibles à la diffusion non-en avant du spin dans les conditions de la diffraction dynamique. On considère les cas des interactions spin-orbite et des interactions hypothétiques qui violent $\mathrm{P}$ et $\mathrm{T}$.

Abstract - The generation of neutron optical effects, which are sensitive to non-forward spin scattering in dynamical diffraction conditions, is studied. Cases of spin-orbit and of supposed $P$ and $T$ violating interactions are considered.

In the study of low-energy neutron spin interactions, the most sensitive experiments are based on neutron optical effects. We consider, for example, the Pviolation effects, namely the spin rotation and the helicity-dependent transmission due to weak currents in the neutron nuclear interaction, which have been obse rved with various nuclear targets and are reviewed in different papers of this Workshop. Effects of the first and of the second kind are determined, respectively, by the real and by the imaginary spin-dependent part of the optical potential. In ordinary targets, the optical potential is proportional to the forward coherent scattering factor $F_{O}$ of target centres, thus excluding the possibility of optical effects related to spin-orbit and to other spin interactions, violating both the $P$ and $T$ symmetry, which do not contribute to forward scattering.

In a perfect crystal, instead, the propagation of coherent neutron waves, approaching diffraction conditions $\underline{k}_{\mathrm{O}}-\underline{\mathrm{k}}_{\mathrm{h}}=\underline{\mathrm{h}}$ (reciprocal lattice vector), is strongly dependent on the periodical potential structure, which appears in the expression of the wave vectors in the combination $F_{h} F_{-h}=F F$ of the reflection and antireflection structure factors. Therefore, coherent spin effects related to non-forward spin scattering are possible and can be described by the dynamical theory of diffraction. Different aspects and cases are considered in a previous work/1/ (and references therein), looking at spin effects in the emergent transmitted and reflected neutron beams. We shortly review a few relevant points.

The scattering amplitudes, for the considered spin interactions, are antisymmetric: $f_{o}\left(\underline{k}, \underline{k}^{\prime}\right)=-f_{o}\left(\underline{k}^{\prime}, \underline{k}\right)$. In this case, a non-monoatomic acentric structure is necessary to have spin terms in $F \bar{F}$. Assuming a complex $f_{\sigma}=\left(s^{\prime}+i s^{\prime}\right) \sigma_{3}$ and a real nuclear amplitude $f_{n}$, and separating the contributions of each amplitude term, we have

$$
F=\left|F_{n}\right| \exp i \delta_{n}+\left|F_{\sigma}\right| \exp i \delta_{\sigma}+i\left|F_{\sigma}^{\prime}\right| \exp i \delta_{\sigma^{*}}^{\prime}
$$

The relevant spin terms, referring the relative phase angles to $\delta_{\mathrm{n}}=0$, are given by

$$
\operatorname{Re}(\mathrm{FF}) \sigma /\left|\mathrm{F}_{\mathrm{n}}\right|=-2\left|\mathrm{~F}_{\sigma}^{\prime}\right| \sin \delta_{\sigma}^{\prime}, \operatorname{Im}(\mathrm{FF})_{\sigma} /\left|\mathrm{F}_{\mathrm{n}}\right|=2\left|\mathrm{~F}_{\sigma}\right| \sin \delta_{\sigma}
$$

and produce, respectively, spin rotation and spin transmission effects. The role of real and imaginary amplitudes is exchanged, with respect to ordinary optics, 
and the magnitude of spin effects is essentially independent of $\left|F_{n}\right|$.

The electromagnetic spin-orbit interaction gives a pure imaginary amplitude $f_{S} \propto$ i $\mu \underline{\sigma} . \underline{k} \times \underline{k}^{\prime} F_{e}$, with $F_{e}$ the electric atom form factor. Related spin rotation effects can be quite large, for example rotation angles per $\mathrm{cm} \mathrm{a} 1 \sim 1$, in the case of quartz and, therefore, could offer a realistic possibility to start an experimental investigation of dynamical spin effects with perfect and non-perfect crystals.

The nuclear spin-orbit is usually negligible, but can be enhanced, with a complex amplitude, in the presence of near-threshold p-resonances. Referring to known nuclear parameters, but to hypothetical crystal samples, we estimate spin rotations $a_{1} \simeq 10^{-2} \sim 10^{-3}$ and polarization transmission effects per $\mathrm{cm} P=10^{-3} \sim$ 10-4. A direct determination of these p-wave effects at threshold, with particular nuclei, may be interesting in connection with $P$-violation experiments.

A simultaneous violation of $P$ and $T$ in the neutron-nucleon interaction would allow a term $f_{P T} \propto i G_{P T} \underline{g}\left(\underline{k}-\underline{k}^{\prime}\right)$ in nuclear scattering $/ 2 /$. In the vicinity of a $p-r e s o-$ nance, $f P T$ is expected to be strongly enhanced and complex, in analogy with $P$ odd $\mathrm{T}$-even interactions $/ 3 /$. We may also refer to known enhanced $P$-violation effects and to assumptions on the relative coupling strength $G_{P T} / G_{P} / 2,4 /$, for an indicative upper limit estimate: $a_{1} \lesssim 10^{-6}$ for thermal neutrons and $a_{1} \approx P_{1} \leqslant 10^{-4}$ at resonance, in agreement with the estimate for another kind of $P$ - and $T$-violation effects, at the $0.75 \mathrm{eV}$ p-resonance of $139 \mathrm{La} / 4 /$.

A most advanced investigation of $P$ and $T$ violation in fundamental interactions is the search for a neutron electric dipole moment, in free-neutron resonance experiments reported in this Workshop. The neutron atomic scattering includes an EDM amplitude of the form $f_{E D M} \propto i \mu_{e} \underline{g}\left(\underline{k}-\underline{k}^{\prime}\right) F_{e}$. An evaluation of the related spin rotation in a quartz crystal gives, for favourable reflections, a 1 rad $/ \mathrm{cm}) \approx 1019 \ell$ with $\ell(\mathrm{cm})=\mu_{\mathrm{e}} / \mathrm{e}$. A conceivable EDM experiment should be based on a backreflection configuration, in the Bragg case, to suppress spin-orbit effects. Particular techniques and feasibility problems are discussed in $/ 1 /$.

Also for the possibility of an experimental investigation of relatively large effects, a crucial problem is the existence of a suitable crystal target. Acentric structures are not so rare and, in a few cases, crystals of good quality are available including a variety of nuclides, however, a question to be first investigated concerns the degree of perfection which is really critical to observe dynamical spin effects.

In the next part of this work we analzse the generation of spin effects inside a perfect crystal. Certain parameters are defined in the usual way by boundary conditions which refer to a plane plate of thickness d, however we will put into evidence rather general relations and results, relevant for a further investigation of spin effects in non-perfect crystals.

In the vicinity of a reflection, $\underline{\mathrm{k}}_{\mathrm{O}}-\underline{\mathrm{k}}_{\mathfrak{n}}=\underline{\mathrm{h}}$, we consider the plane-wave solution in a periodical potential

$$
\begin{aligned}
\psi= & \varphi_{0}+\varphi_{h}, \varphi_{o, h}=\left[\left(A_{o, h}\right)_{+} \exp \left(i \bar{Z}_{+} \bar{g}_{o} k \hat{n} \cdot \underline{r}\right)+\left(A_{o, h}\right)_{-} \exp \left(i \bar{Z}_{-} \bar{g}_{0} k \hat{n} \cdot \underline{r}\right)\right] . \\
& \exp \left(i \underline{k}_{0}, h \cdot \underline{r}\right)
\end{aligned}
$$

where $g_{0}=1 / \bar{g}_{0}=\underline{\hat{k}}_{0} \cdot \hat{n}$ and $A_{h \pm} / A_{0 \pm}=-Z_{\underline{t}} / F$.

Continuity conditions at the entrance surface, with an incident wave $\varphi_{k}=\exp (\mathbf{i k} . \underline{r})$, determine the direction of $\hat{n}$, parallel to the ingoing normal, and $\underline{k}_{\mathrm{o}}=\underline{\mathrm{k}}+(1 / 2) \mathrm{k} \mathrm{F}_{\mathrm{og}} \hat{\mathrm{n}}$ (ordinary refraction, taking $F_{\mathrm{o}}$ and $\mathrm{F}$ in units of $\mathrm{k}^{2}$ ).

The phase velocities have the form 


$$
\overline{\mathrm{Z}}_{\dot{ \pm}}=-\mathrm{X} \pm \mathrm{W} \text { with } \mathrm{X}=\mathrm{g}\left(\underline{\mathrm{k}}_{\mathrm{o}}-\underline{\mathrm{k}}_{\mathrm{B}}\right) \cdot \underline{\mathrm{hk}}^{-2}=\mathrm{g}\left(\mathscr{E}-\mathscr{E}_{\mathrm{B}}\right), \mathrm{W}=\left(\mathrm{X}^{2}+\mathrm{gF} \overline{\mathrm{F}}\right) \mathrm{l} / 2
$$

Here $\mathrm{X}$, with $2 \mathrm{kB} \cdot \underline{\mathrm{h}}=\mathrm{h}^{2}$, represents the deviation from the centre of the diffraction line, or resonance error, and $W$ is the pendellösung frequency, while the reflection geometry determines $g_{h}=\hat{k}_{h}$. $\hat{n}$ and $g=1 / \bar{g}=g_{0} / g_{h}$. It is convenient to separate the nucleax and the much smaller spin term, writing $\left.\bar{F} \overline{\mathrm{F}}=(\mathrm{FF})_{\mathrm{n}}+\mathrm{FF}\right)_{\sigma}$, with $(F F)_{n} \approx\left|F_{n}\right|^{2}$ (assuming a negligible absorption) and to introduce reduced units.

$$
x=x /\left|F_{n}\right|, w=w /\left|F_{n}\right|, z_{ \pm}=x \pm w, t=k\left|F_{n}\right| \hat{n}, \underline{r}
$$

where $t$ is the penetration depth in extinction length units, putting $t=0$ at the entrance surface. The spin effects are essentially due to spin terms in the exponential factors of (2), given by a reduced form (where $\bar{g}_{0} t$ is a coordinate along $\hat{k}_{0}$ )

$$
\exp \left(i Z_{ \pm} \bar{g}_{0} \mathrm{t}\right), \mathrm{z}_{ \pm}=-\mathrm{x} \pm\left(\mathrm{w}_{\mathrm{n}}+\mathrm{w}_{\sigma}\right), \quad \mathrm{w}_{\sigma} \approx\left(\mathrm{g} / 2 \mathrm{w}_{\mathrm{n}}\right)(\mathrm{FF})_{\sigma} /\left|\mathrm{F}_{\mathrm{n}}\right|^{2}
$$

In other cases we can safely approximate

$$
\mathrm{A}_{\mathrm{h} \pm} / \mathrm{A}_{\mathrm{o \pm}} \approx-\mathrm{z}_{ \pm} ; \mathrm{w} \approx \mathrm{w}_{\mathrm{n}} \approx\left(\mathrm{x}^{2}+\mathrm{g}\right)^{1 / 2} ; \mathrm{z}_{+} \mathrm{z}_{-} \approx-\mathrm{g}
$$

In the Bragg case, we implicitly exclude the range where $\mathrm{w}^{2} \leqslant 0$ (no transmission). The amplitude ratio is determined by the boundary conditions $\varphi_{h}(0)=0$ in the Laue case and $\varphi_{h}(d)=0$ in the Bragg case, obtaining respectively

$$
A_{o \pm}=\mp z_{\mp} /\left(z_{+}-z_{-}\right) \text {and } A_{o \pm}=\mp z_{\mp} \exp \left(\bar{f}_{i w g_{0}} d\right) / N
$$

with $\mathrm{N}$ the sum of numerators, to have $\mathrm{A}+\mathrm{A}_{--}=\varphi(0)=\varphi_{\mathrm{k}}(0)=1$. Therefore, in the Bragg case, the wave amplitudes include a pendellösung factor, with an effective dependence on spin terms.

The relation $\left|A_{0}+\right|^{2} /\left|A_{0}\right| 2 \approx z^{2} / z^{2}$ means that, for a sufficiently large resonance error (say for $w \geqslant \mathrm{~g}^{1 / 2}$ in the Bragg case) and corresponding to its sign, there is one dominant transmitted wave, associated with the smaller of $\left|z_{+}\right|$. A qualitative validity of this result can be stated, in general, by the physical assumption $\left|A_{h+}\right|^{2}+\mid\left(\left.A_{h-}\right|^{2}<\left|A_{o+}\right|^{2}+\left|A_{0-}\right|^{2}\right.$.

To proceed, it is convenient to consider the current densities associated with $\varphi_{\mathrm{o}}(\underline{\mathrm{r}})$ and $\varphi_{\mathrm{h}}(\underline{\mathrm{r}})$, given by $\underline{\mathrm{I}}_{\mathrm{o}, \mathrm{h}}(\underline{\mathrm{r}})=\underline{\mathrm{k}}_{\mathrm{O}, \mathrm{h}}\left(\varphi_{\mathrm{O}}^{*} \mathrm{~h} \varphi_{\mathrm{O}}\right)$. By means of $(2)$, dis regarding the spin dependence, for the moment, and putting $k\left|A_{o}\right|^{2}=I_{0 \pm}, k\left|A_{h \pm}\right|^{2}=I_{h \pm}$, $\mathrm{z}_{\underline{1}}^{2}=\mathrm{R}_{ \pm}$, we obtain the flux density, for any $\mathrm{I}_{0+} / \mathrm{I}_{\mathrm{O}_{-}}$,

$$
\begin{aligned}
\phi(t)= & \underline{J} \cdot \hat{\mathrm{n}}=\phi_{\mathrm{o}}+\phi_{\mathrm{h}}=\mathrm{J}_{\mathrm{o}} \mathrm{g}_{\mathrm{o}}+\mathrm{J}_{\mathrm{h}} \mathrm{g}_{\mathrm{h}}=\mathrm{g}_{\mathrm{o}}\left(\mathrm{I}_{\mathrm{o}}+\mathrm{I}_{\mathrm{o}-}\right)+\mathrm{g}_{\mathrm{h}}\left(\mathrm{I}_{\mathrm{h}}+\mathrm{I}_{\mathrm{h}-}\right)= \\
& \mathrm{go}_{\mathrm{o}}\left[\mathrm{I}_{\mathrm{o}}+\left(1+\overline{\mathrm{g}} \mathrm{R}_{+}\right)+\mathrm{I}_{\mathrm{o}-}\left(1+\mathrm{g}_{-}\right)\right]
\end{aligned}
$$

With our boundary conditions, a simple algebra determines the splitting ratio of the incident flux $\phi_{\mathrm{k}}=\mathrm{kg}_{\mathrm{o}}$ into $\phi_{\mathrm{o}}$ and $\phi_{\mathrm{h}}$ at the surfaces, reproducing well known results. In the Bragg case, referring to pendellösung averages $\left\{I_{0 \pm}\right\}=R_{\mp} /\left|R_{+}-R_{-}\right|$ we obtain $\{\phi(t)\}=\left\{\varphi_{0}(d)\right\}=k g o w / x$ (outgoing transmitted flux).

Extending this formalism, we define spin current densities, regarding $\varphi_{\mathrm{O}, \mathrm{h}}$ as space $\mathrm{x}$ spin functions:

$$
\left.\left(\underline{S}_{0}, h\right)_{i}=\underline{S}_{0, h}=\underline{k}_{0}, h<\varphi_{0}, h\left|\sigma_{i}\right| \varphi_{0, h}\right\rangle_{0} \quad ; \underline{S}_{i}=\underline{S}=\underline{S}_{0}+\underline{S}_{h}
$$

where i may be omitted. We first describe a small spin transmission effect, due to $\mathrm{w}_{\sigma}=i b \sigma_{3},(|b d| \ll<1)$. To evaluate the flux $\hat{n} . \underline{S}_{3}$ of $\sigma_{3}$, we have to take the difference of $\underline{\mathrm{J}} . \hat{\mathrm{n}}$ in the spin states $\sigma_{3}= \pm 1$. The result 


$$
\hat{n} \underline{S}(t)=-2 b \bar{g}_{0} t\left[g_{0}\left(I_{o+}-I_{o-}\right)+g_{h}\left(I_{h+}-I_{h-}\right)\right]+\hat{n} \cdot \underline{S}(0)=\Sigma+\hat{n} \cdot \underline{S}(0)
$$

shows a linear growth of the spin flux versus $t$, while the initial value $\hat{n} \underline{S}(0)=$ $\hat{n} S_{h}(0)$, for $S_{o}(0)=0$ (unpolarized incident beam) is the negative of the outgoing spin flux of the reflected beam, in the Bragg case, and is vanishing, in the Lane case. In simple words, in (7), the linear term $\Sigma$ represents the total neutron spin generated by the interaction and carried to the surfaces by the transmitted and reflected beams, while the constant term is due to the inherent spin depen-

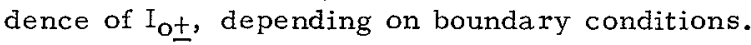

The equivalent form $\quad \Sigma=-2 b t\left[I_{O^{+}}\left(1+\bar{g} R_{+}\right)-I_{O}\left(1+\bar{g} R_{-}\right)\right]$allows to verify that, in the Bragg case, on either side of the transmission curve, (that is for $-\bar{g} R_{+}<I$ and $-\overrightarrow{g R} R_{-}>1$ or viceversa) the sign of $\Sigma$ is uniquely defined and reverses like $x$. This relevant result is valid in general.

Our boundary conditions give $\mathrm{I}_{\mathrm{h}+}=\mathrm{I}_{\mathrm{h}-}$ and, therefore, $\Sigma=-2 \mathrm{bt}\left(\mathrm{I}_{\mathrm{o}} \mathrm{f}_{-} \mathrm{I}_{\mathrm{O}_{-}}\right)$. In the Bragg case, the pendellösung average is $\{\Sigma\}=-2 b t \mathrm{x} /|\mathrm{x}|$. In the Lave case $\Sigma=\hat{n} . \underline{S}=\hat{n}_{S}$, with no spin effect in $J_{h}$ (with no nuclear absorption).

Finally, we can evaluate the neutron flux polarization, given by

$$
P(t)=\hat{n} \cdot \underline{S}(t) / \hat{n} \cdot \underline{I}=\Sigma / \phi+\hat{n} \cdot \underline{S}(0) / \phi
$$

In the Bragg case, where $\mathrm{P}(\mathrm{d})$ is the transmitted beam polarization, we have

$$
\left\{P_{3}(t)\right\}=-2 b \bar{g}_{0} t(x / w)-b \bar{g}_{0} d(w / x-x / w) ;\left\{P_{3}(d)\right\}=-b \bar{g}_{0} d(x / w+w / x)
$$

In $\left\{\mathrm{P}_{3}(t)\right\}$, only the constant term is an average. According to (9) and (4), some enhancement of spin effects (not included in our previous estimates) is expected near the total reflection edge $(\mathrm{w} \rightarrow 0)$ and in asymmetric reflection with $|\mathrm{g}| \gg 1$, in the Laue case too.

To describe the spin rotation a round $\hat{x}_{3}$, due to $\mathrm{w}_{\sigma}=\mathrm{a} \sigma_{3}$, with $\mid$ ad $\mid \ll 1$, we may consider an incident beam completely polarized along $\hat{x}_{1}$. The same formal relations a re obtained but with $-a, S_{2}, P_{2}$, etc., in place of $b, S_{3}, P_{3}$, etc. The approximate spin rotation is then given by $\mathrm{P}_{2}$.

For a sufficiently large resonance error and a relatively low reflection intensity, the re is a predominant $\mathrm{I}_{\mathrm{O}}$ or $\mathrm{I}_{\mathrm{O}_{-}}$, with a spin phase-velocity $+\mathrm{w}_{\sigma}$ or $-\mathrm{w}_{\sigma}$, corresponding to the error sign. In this approach, by dividing ( 7 ) by (5), we obtain the simple relation $\mathrm{P}_{3} \approx-2 \mathrm{bg}_{\mathrm{o}} \mathrm{t}(\mathrm{x} /|\mathrm{x}|)$, where $\mathrm{b} \propto \mathrm{gw}^{-1}$, and the analogous for $\mathrm{P}_{2}$. In general, in the limit $\mathrm{X}^{2}>\mathrm{gFF}$, that is $\mathrm{W} \approx \mathrm{X}=\mathrm{g}\left(\mathscr{E}-\mathscr{E}_{\mathrm{B}}\right)$, a solution (2) reduces to one refracted wave propagating in a direction near $\hat{k}_{0}$, in a spin-dependent optical potential $(\mathrm{F} \overline{\mathrm{F}})_{\sigma} / 2\left(\mathscr{E}-\mathscr{E}_{\mathrm{B}}\right)$. This form, independent of boundary conditions, represents the effective dynamical or "resonance" neutron spin interaction with the crystal.

A relevant question concerns the existence of coherent spin effects in non-perfect crystals, where the optical-potential periodicity is altered. In this case, simple plane wave solutions like (2) do not apply, but may be replaced following Takagi $/ 5,6 /$, by wave packets with a space representation

$$
\psi(\underline{r})=D_{0}(\underline{r}) \exp \left(i \underline{k}_{0} \cdot \underline{r}\right)+D_{n}(\underline{r}) \exp \left(i \underline{k}_{h} \cdot r\right)
$$

where $D_{0}(\underline{r}), D_{h}(\underline{r})$ are (narrow) distribution functions around refracted and reflected neutron trajectories, which satisfy partial differential equations of the kind

$$
\left(\partial^{2} / \partial q_{0} \partial q_{h}\right) D_{0}(\underline{r})-i k X^{\prime}\left(\partial / \partial q_{o}\right) D_{o}(\underline{r})+k^{2} F \bar{F} D_{o}(\underline{r})=0
$$


where qo, $q_{h}$ are coordinates along $\hat{k}_{0}, \hat{k}_{h}$. The lattice deformation, as a function of position, appears in $X^{\prime}=X-k^{-1}\left(\partial / \partial q_{h}\right) \underline{h} . \underline{\underline{u}}(\underline{r})$, where $\underline{u}(\underline{r})$ is the displacement of the atoms of a unit cell (regarded as rigid) with respect to a perfect reference lattice, for which $X$ is defined. Usually, the second term in $X^{\prime}$ is as sumed relatively small and slowly varying. In regions with no distortion, the Takagi equations are satis fied by a packet of ordinary solutions (2). As a first approach we consider the case of a back reflection, in the Bragg case, in a system composed of quasi-perfect blocks of a sufficient size (at least several estinction lengths) with tilt angles, relative to the reference lattice, definitely less than the intrinsic width of the reflection ( $\sim$ minutes). Therefore, in any block, $g=-1$ and $X^{\prime}(\underline{r}) \approx X$ (reference value, determined by $\mathrm{k}$ ). For neutrons transmitted on a given side of the transmission curve, a substantial spin effect will result by the accumulation of regular increments in each block while weak irregular spin changes through grain boundaries may be disregarded.

The influence of lattice distortion can be studied by eq. (11) with $\left(\partial / \partial q_{h}\right)=-\left(\partial / \partial q_{0}\right)$, which may be rewritten in reduced units $t=k\left|F_{n}\right| q_{0}, x^{\prime}=X^{1} /\left|F_{n}\right|$, etc. The equation admits a double solution $t D_{0}(\underline{x})$. It is easily seen that, by introducing a small spin term in $\mathrm{F} \overline{\mathrm{F}}=\left|\mathrm{F}_{\mathrm{n}}\right|^{2}+(\mathrm{F} \overline{\mathrm{F}})_{\sigma}=\left|\mathrm{F}_{\mathrm{n}}\right|^{2}\left(1+\mathrm{c} \sigma_{3}\right)$, the solution is varied, approximately, by a factor $\exp \left[\mathrm{i} \sigma_{3} \delta_{ \pm}(\mathrm{t})\right]$ (for $\left.\delta_{ \pm} \ll 1\right)$, such that

$$
\delta_{ \pm}\left(t^{\prime \prime}\right)-\delta_{ \pm}\left(t^{\prime}\right) \approx \int_{t^{\prime}}^{t^{\prime \prime}}\left( \pm \mathrm{c} / 2 w^{\prime}\right) d t, \text { with } w^{\prime}=\left(x^{\prime 2}-1\right)^{1 / 2}
$$

Therefore, also in non-periodical solutions $D_{0 \pm}$, respective spin-dependent phasefactors can be separated (with $\delta+(t)$ depending on the neutron trajectory) and spin coherence is preserved. This simple extension of the perfect-lattice case (where $\mathrm{w}^{\prime}(\underline{x})=\mathrm{w}$ and $c / 2 \mathrm{w}^{\prime}=\mathrm{a}$ or $\left.\mathrm{ib}\right)$ specifies the qualitative description of spin effects in the previous picture, and allows to foresee qualitatively similax effects in dis torted blocks and in the whole crystal. Indeed, with reasonable physical assumptions, further considerations following the line of previous cases, support the sign coherence of spin effects along neutron trajectories, when the transmission occurs on one side of the effective reflection curve (including distortion and mosaic broadening and assuming no satellites). In particular, a description with one wave-packet (either $\mathrm{D}_{\mathrm{o}^{+}}, \delta_{+}$or $\mathrm{D}_{\mathrm{O}_{-}}, \delta_{-}$) is meaningful in the limit of a good trans mission in analogy with the perfect crystal case.

In summary, the existence of coherent spin effects does not necessarily require a long-range coherence of neutron waves but, at least a sign coherence of the differential spin-dependent phase velocity (or attenuation) along the neutron trajectories.

The present discussion is preliminary, and does not consider, for instance, the role of waves reflected and diffused by crystal imperfections. Besides the exact back-reflection, other cases should be considered. Finally, we suggest that an alte rnative mechanism for the selection of neutron waves with univocal spin effects is naturally occurring in (moderately) absorbing crystals and, particularly, in the anomalous transmission (also in the reflection) in the Laue case.

\section{References}

/1/ FORTE M., Conf. Neutron and its Application, Cambridge (1982) Contr. 4. B. 4 and J. Phys. G. 2 (1983) 745.

/2/ SZYMANSKI Z., Nucl. Phys. A 113 (1968) 385.

13/ FORTE M., Fundamental Physics with Reactor Neutron and Neutrinos (1977) Inst. Phys. Conf. Ser. 42 (1978).

/4/ BUNAKOV V.E. and GUDKOV V. P., Leningrad, Nucl. Phys. Inst. Preprint 777 (1982).

/5/ TAKAGI S., Acta Cryst. 23 (1962) 23.

/6/ TAKAGI S., J. Phys. Japan, 26 (1969) 1239. 